\title{
DETERMINATION OF THE WRENCH-CLOSURE WORKSPACE OF 6-DOF PARALLEL CABLE-DRIVEN MECHANISMS
}

\author{
M. Gouttefarde, J-P. Merlet and D. Daney \\ INRIA Sophia-Antipolis, France \\ Marc.Gouttefarde@sophia.inria.fr \\ Jean-Pierre.Merlet@sophia.inria.fr \\ David.Daney@sophia.inria.fr
}

\begin{abstract}
The wrench-closure workspace of parallel cable-driven mechanisms is the set of poses of their mobile platform for which the cables can balance any external wrench. The determination of this workspace is an important issue since the cables can only pull and not push on the mobile platform. This paper deals with the wrench-closure workspace of six-degrees-of-freedom (DOF) parallel mechanisms driven by $m$ cables, $m \geq 7$. The boundary of the constant-orientation cross sections of the wrench-closure workspace is shown to consist of parts of cubic surfaces and an efficient method that determine this workspace by delineating its boundary is proposed.
\end{abstract}

Keywords: Cable-driven mechanism, parallel mechanism, wrench-closure, workspace, Gough-Stewart platform.

\section{Introduction}

A parallel cable-driven mechanism consists essentially of a mobile platform connected in parallel to a base by light weight links such as cables. The control of the length of the cables allows the control of the pose of the platform. For instance, a mechanism driven by eight cables is shown in Fig. 1. Parallel cable-driven mechanisms have several advantages over conventional rigid-link mechanisms (Barrette and Gosselin, 2005, Merlet, 2004, Roberts et al., 1998). The mass and inertia of the moving part is reduced and they are less expensive. Moreover, parallel cable-driven mechanisms are easier to build, transport and reconfigure and they have the possibility of working in a very large space. Consequently, parallel cable-driven mechanisms have been used in several applications such as, for instance, robotic cranes (Dagalakis et al., 1989), high speed manip- 


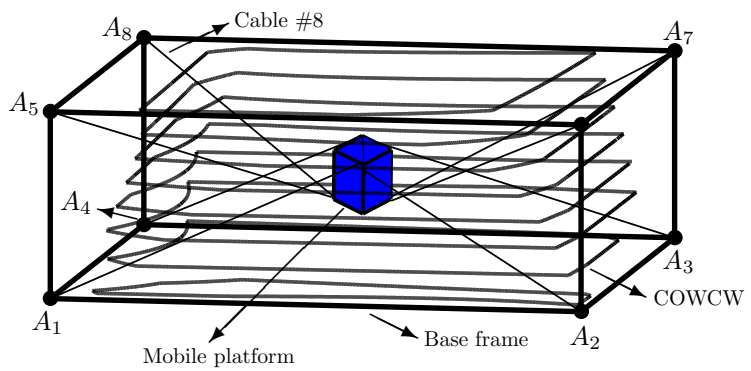

Figure 1. A 6-DOF Parallel Mechanism Driven by Eight Cables and its ConstantOrientation Wrench-Closure Workspace

ulation (Kawamura et al., 2000), active suspension devices (Lafourcade, 2004) and virtual reality (Merlet, 2004).

This paper deals with the determination of the workspace of six-DOF parallel cable-driven mechanisms. This workspace may be limited by the total length of each cable, by the interferences between the cables and between the cables and the mobile platform and by the unidirectional nature of the forces applied by the cables on the mobile platform. The limitations due to the total lengths of the cables can be determined by means of algorithms presented in (Gosselin, 1990) and in (Merlet, 1999). However, the workspace will usually not be limited by the total lengths of the cables since large total lengths can generally be used. For a constant orientation of the mobile platform, the problem of the influence on the workspace of the cables interferences is addressed in (Merlet, 2004). The third limitation which is due to the unidirectional nature of the forces applied by the cables on the platform has been studied mainly in the case of planar parallel cable-driven mechanisms in (Barrette and Gosselin, 2005, Fattah and Agrawal, 2005, Gallina and Rosati, 2002, Gouttefarde and Gosselin, 2006, Roberts et al., 1998, Stump and Kumar, 2004, Verhoeven and Hiller, 2000, Verhoeven, 2004, Williams et al., 2003).

All these references do not share the same definition of the workspace and two different types of workspace can be distinguished. Indeed, we can study the set of poses of the mobile platform for which the cables can balance, by pulling on the mobile platform,

- a particular wrench or a particular set of wrenches, or

- any wrench.

When the first type of workspace is to be determined, the particular wrench or the particular set of wrenches must be chosen. Hence, the de- 
termination of this workspace is useful when a particular task, for which the wrenches that must be generated at the mobile platform are known, is assigned to the cable-driven mechanism. The determination of the second type of workspace, called the wrench-closure workspace (WCW) in the present paper, does not need such a choice since it depends on the geometry of the mechanism only (Gouttefarde and Gosselin, 2006), i.e., on the number of cables, on the positions of the attachment points of the cables at the mobile platform and on the positions of the points of the base from which the cables are taut (points $A_{i}$ in Fig. 1). Hence, the WCW is a property of the mechanism only and is consequently related to the general design problem of selecting geometry of mechanisms which have the ability to perform various types of tasks. More details on the different types of workspace of parallel cable-driven mechanisms can be found in (Bosscher and Ebert-Uphoff, 2004, Ebert-UpHoff and Voglewede, 2004, Gouttefarde and Gosselin, 2006, Gouttefarde, 2005).

In this paper, an efficient method that allows to determine the constantorientation cross sections of the WCW, called the constant-orientation WCW (COWCW), is proposed. This method applies to any six-DOF parallel cable-driven mechanism and it determines the boundary of the COWCW which is shown to consist of parts of cubic surfaces. To the best of our knowledge, such a tool has never been introduced in previous papers dealing with six-DOF cable-driven mechanisms such as the works presented in (Hiller et al., 2005, Kawamura et al., 2000, Lafourcade, 2004, Pham et al., 2006, Tadokoro et al., 1996, Takeda and Funabashi, 2000, Verhoeven, 2004).

\section{Nature of the boundary of the COWCW}

The relationship between the tensions in the cables and the wrench $\mathbf{w}_{p}$ applied by the cables on a reference point $P$ of the platform is given by

$$
\mathbf{W} \mathbf{t}=\mathbf{w}_{p}
$$

where $\mathbf{t}$ is the vector of cable tensions and $\mathbf{W}$ the $6 \times m$ pose dependent wrench matrix where $m$ denotes the number of cables. Since the WCW of a six-DOF parallel cable-driven mechanism exists only if its mobile platform is driven by at least seven cables (Ming and Higuchi, 1994), in this paper, $m \geq 7$.

The WCW is defined as the set of poses of the mobile platform in which, for any wrench $\mathbf{w}_{p}$ in $\mathbb{R}^{6}$, there exists at least one vector $\mathbf{t}$ in $\mathbb{R}^{m}$, whose components are all nonnegative, such that Eq. 1 is verified. For 
a constant orientation of the mobile platform, the COWCW is the set of positions of the reference point $P$ of the platform which belongs to the WCW. The poses that belongs to the WCW can be characterized by several means (Gouttefarde, 2005) including the following theorem ( Murray et al., 1994, Stump and Kumar, 2004).

Theorem 1 Let $\mathbf{w}_{i}, 1 \leq i \leq m, m>6$, be the column vectors of the wrench matrix $\mathbf{W}$ and let us assume that $\operatorname{rank}(\mathbf{W})=6$. Then, a pose of the mobile platform belongs to the $W C W$ if and only if all the hyperplanes $\mathcal{H}$ of $\mathbb{R}^{6}$ spanned by five wrenches $\mathbf{w}_{i}$ (linearly independent) are separating, i.e.,

$$
\exists(j, k), j \neq k, \text { such that } \mathbf{w}_{j}^{T} \mathbf{q}>0 \text { and } \mathbf{w}_{k}^{T} \mathbf{q}<0,
$$

where $\mathbf{q} \neq \overrightarrow{\mathbf{0}}$ is a vector of $\mathbb{R}^{6}$ orthogonal to $\mathcal{H}$.

Eq. 2 means that there exists at least one column of $\mathbf{W}$ lying on each side of the hyperplane $\mathcal{H}$.

Now, let us assume that the orientation of the mobile platform of a six-DOF parallel cable-driven mechanism is constant. Let us consider a position $P_{1}$ of the mobile platform which belongs to the COWCW and a continuous trajectory going from $P_{1}$ to a position $P_{2}$ which lies outside the COWCW. According to Theorem 1, on the part of the trajectory that belongs to the COWCW, all the hyperplanes $\mathcal{H}$ spanned by five columns of the wrench matrix $\mathbf{W}$ are separating. Moreover, at the point at which the trajectory leaves the COWCW, at least one of the hyperplane $\mathcal{H}$ ceases to be a separating hyperplane. But, a hyperplane $\mathcal{H}$ ceases to be a separating hyperplane if and only if the five columns of $\mathbf{W}$ which span $\mathcal{H}$ become linearly dependent or these five columns and an other column of $\mathbf{W}$ become linearly dependent. Hence, in the two cases, the trajectory leaves the COWCW since at least one combination of six columns of $\mathbf{W}$ becomes linearly dependent and a position of the mobile platform belongs to the boundary of the COWCW only if six columns of the wrench matrix $\mathbf{W}$ are linearly dependent.

This observation on the nature of the boundary of the COWCW is important. Indeed, the columns of the wrench matrix $\mathbf{W}$ are of the same nature as the columns of the transpose of the so-called jacobian matrix of a Gough-Stewart platform (Ebert-UpHoff and Voglewede, 2004, Gouttefarde and Gosselin, 2005) and, consequently, for a constant orientation of the mobile platform, six columns of $\mathbf{W}$ are linearly dependent on a surface of the same nature as the constant-orientation singularity locus of a Gough-Stewart platform which is known to be a cubic surface 
(Mayer St-Onge and Gosselin, 2000), i.e., a surface whose equation can be written as a multivariate polynomial of degree three in the Cartesian coordinates of the mobile platform. Finally, we can state that the boundary of the COWCW consists of parts of cubic surfaces of the same nature as the constant-orientation singularity locus of the Gough-Stewart platform. This fundamental result is a generalization to the most general case of the similar result presented in (Gouttefarde and Gosselin, 2005) in the case of six-DOF parallel mechanisms driven by seven cables.

\section{Efficient determination of the COWCW}

In this section, the main steps of an efficient method that determines the COWCW by finding its boundary are presented. This method takes advantage of the geometric nature of the boundary introduced in the previous section.

First, an orientation of the mobile platform is chosen and, for each combination of six columns of the wrench matrix $\mathbf{W}$, the equation of the cubic surface $\mathcal{S}_{i}$ on which these columns are linearly dependent is determined. A method that allows to obtain these equations is presented in (Mayer St-Onge and Gosselin, 2000). Then, in order to deal with curves instead of surfaces, a set of parallel planes is selected, e.g. planes orthogonal to the $z$-axis, and the part of the boundary of the COWCW which belongs to these planes is sought. Let us denote by $\mathcal{P}$ one of these planes. Without loss of generality, we can assume that the equation of $\mathcal{P}$ is $z=z_{0}$, i.e., that $\mathcal{P}$ is orthogonal to the $z$-axis. The part of the boundary of the COWCW which lies in $\mathcal{P}$ can be obtained by following the steps presented below.

Step 1: obtain the equations of the cubic curves $\mathcal{C}_{i}$ that lie in $\mathcal{P}$ and on which six columns of $\mathbf{W}$ are linearly dependent by substituting $z_{0}$ for $z$ in the equations of the cubic surfaces $\mathcal{S}_{i}$.

According to section 2, if it exists, the part of the boundary of the COWCW which lies in $\mathcal{P}$ is composed of parts of the cubic curves $\mathcal{C}_{i}$. Moreover, the extremities of these parts are the points of intersection between the cubic curves $\mathcal{C}_{i}$.

Step 2: for each of the cubic curve $\mathcal{C}_{i}$, determine the points of intersection between $\mathcal{C}_{i}$ and the other cubic curves.

The points of intersection between the cubic curves $\mathcal{C}_{i}$ can be determined by means of an elimination method (Roth, 1993).

Step 3: for each of the cubic curve $\mathcal{C}_{i}$, find all its parts defined by the points of intersection computed at step 2 and eliminate all unbounded parts. 


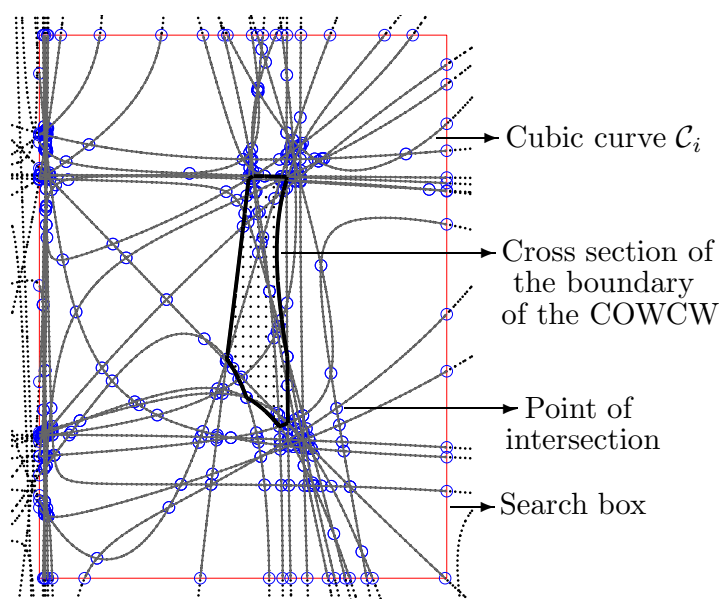

Figure 2. In a plane $\mathcal{P}$, the cubic curves $\mathcal{C}_{i}$ and the boundary of the COWCW

In the present work, the different parts $\mathcal{C}_{i j}$ of a cubic curve $\mathcal{C}_{i}$ are found by following the curve $\mathcal{C}_{i}$ by means of a continuation method ( Merlet, 2004b). The unbounded parts of the curves $\mathcal{C}_{i}$ are eliminated for the COWCW is a bounded subset of $\mathbb{R}^{3}$ (Gouttefarde, 2005).

Step 4: test each of the parts of cubic curves $\mathcal{C}_{i}$ found in step 3 in order to obtain those which, in $\mathcal{P}$, constitute the boundary of the COWCW.

Let $\mathcal{C}_{i j}$ be a part of a curve $\mathcal{C}_{i}$. If one of the points $Q$ of $\mathcal{C}_{i j}$ apart from its two extremities belongs to the COWCW then $\mathcal{C}_{i j}$ is not a part of the boundary since the COWCW is an open set. On the contrary, if $Q$ does not belong to the COWCW, two points $Q_{1}$ and $Q_{2}$ near enough $Q$ are considered. $Q_{1}$ lies on one side of $\mathcal{C}_{i j}$ and $Q_{2}$ on the other side. Then, $\mathcal{C}_{i j}$ is a part of the boundary if and only if $Q_{1}$ or $Q_{2}$ belongs to the COWCW. Note that, in order to determine if a given point belongs to the COWCW, the interior of the convex hull formed by the columns of the wrench matrix $\mathbf{W}$ must contain the origin of $\mathbb{R}^{6}$ (Murray et al., 1994).

Step 5: if some parts of the cubic curves $\mathcal{C}_{i}$ remain after step 4, the COWCW exists and a graphical representation of the part of its boundary which lies in the plane $\mathcal{P}$ is obtained by drawing these parts.

\section{Example}

The COWCW shown in Fig. 1 has been determined, for the orientation of the mobile platform shown in the figure, by following the steps introduced in the previous section. In one of the plane $\mathcal{P}$, the cubic 
curves $\mathcal{C}_{i}$, their points of intersection and the cross section of the boundary of the COWCW obtained by the method are shown in Fig. 2.

\section{Conclusions}

The determination of the WCW is an important issue for 6-DOF parallel cable-driven mechanisms since the cables cannot push on the mobile platform. Moreover, the COWCW turns out to be highly dependent on the geometry of the mechanism and on the orientation of its mobile platform. Hence, the method presented in this paper which allows to determine the COWCW is useful in order to analyze and design 6-DOF parallel cable-driven mechanisms. This method applies to 6-DOF mechanisms driven by an arbitrary number of cables and is based on the nature of the boundary of the COWCW which has been shown to consist of parts of cubic surfaces of the same nature as the constant-orientation singularity locus of Gough-Stewart platforms.

\section{References}

Barrette, G., and Gosselin, C. M. (2005), Determination of the dynamic workspace of cable-driven planar parallel mechanisms, Journal of Mechanical Design, vol. 127, no. 2, pp. 242-248.

Bosscher, P., and Ebert-Uphoff, I. (2004), Wrench-based analysis of cable-driven robots, IEEE International Conference on Robotics and Automation, New Orleans, LA, USA.

Dagalakis, N. G., Albus, J. S., Wang, B.-L., Unger, J., and Lee, J.D. (1989), Stiffness study of a parallel link robot crane for shipbuilding applications, Journal of Offshore Mechanics and Arctic Engineering, vol. 111, pp. 183-193.

Ebert-UpHoff, I., and Voglewede, P. A. (2004), On the connections between cabledriven robots, parallel manipulators and grasping, IEEE International Conference on Robotics and Automation, New Orleans, LA, USA.

Fattah, A., and Agrawal, S. K. (2005), On the design of cable-suspended planar parallel robots, Journal of Mechanical Design, vol. 127, pp. 1021-1028.

Gallina, P., and Rosati, G. (2002), Manipulability of a planar wire driven haptic device, Mechanism and Machine Theory, vol. 37, no. 2, pp. 215-228.

Gosselin, C. M. (1990), Determination of the workspace of 6-DOF parallel manipulators, Journal of Mechanical Design, vol. 112, no. 3, pp. 331-336.

Gouttefarde, M. (2005), Analyse de l'espace des poses polyvalentes des mécanismes parallèles entraînés par câbles, Ph.D. Dissertation, Laval University, Québec, Canada.

Gouttefarde, M., and Gosselin, C. M. (2006), Analysis of the wrench-closure workspace of planar parallel cable-driven mechanisms, IEEE Transactions on Robotics, to appear.

Gouttefarde, M., and Gosselin, C. M. (2005), Wrench-closure workspace of six-dof parallel mechanisms driven by 7 cables, CCToMM Symposium on Mechanisms, Machines, and Mechatronics, Montréal, Québec, Canada. 
Hiller, M., Fang, S., Mielczarek, S., Verhoeven, R., and Franitza, D. (2005), Design, analysis and realization of tendon-based parallel manipulators, Mechanism and Machine Theory, vol. 40, pp. 429-445.

Kawamura, S., Kino, H., and Won, C. (2000), High-speed manipulation by using parallel wire-driven robots, Robotica, vol. 18, no. 1, pp. 13-21.

Lafourcade, P. (2004), Contribution à l'étude des manipulateurs parallèles à câbles, application à la conception d'une suspension active pour soufflerie, Ph.D. Dissertation, ONERA-DCSD, Toulouse, France.

Mayer St-Onge, B., and Gosselin, C. M. (2000), Singularity analysis and representation of the general Gough-Stewart platform, The International Journal of Robotics Research, vol. 19, no. 3, pp. 271-288.

Merlet, J-P. (2004), Analysis of the influence of wires interference on the workspace of wire robots, in Advances in Robot Kinematics, Sestri-Levante, Italy.

Merlet, J-P. (1999), Determination of 6D workspaces of Gough-type parallel manipulator and comparison between different geometries, The International Journal of Robotics Research, vol. 18, no. 9, pp. 902-916.

Merlet, J-P. (2004b), ALIAS, [Online]. Available: http://www-sop.inria.fr/coprin/logiciels/ALIAS/

Ming, A., and Higuchi, T. (1994), Study on multiple degree-of-freedom positioning mechanism using wires (Part 1) - concept, design and control, International Journal of the Japan Society for Precision Engineering, vol. 28, no. 2, pp. 131-138.

Murray, R., Li, Z., and Sastry, S. (1994), A mathematical introduction to robotic manipulation, CRC Press, Boca Raton, FL, USA.

Pham, C.B., Yeo, S.H., Yang, G., Kurbanhusen, M.S., and Chen, I-M. (2006), Forceclosure workspace analysis of cable-driven parallel mechanisms, Mechanism and Machine Theory, vol. 41, no. 1, pp. 53-69.

Roberts, R. G., Graham, T., and Lippitt, T. (1998), On the inverse kinematics, statics, and fault Tolerance of cable-suspended robots, Journal of Robotic Systems, vol. 15, no. 10 , pp. 581-597.

Roth, B. (1993), Computations in Kinematics, in Computational Kinematics, Dagstuhl Castle, Germany.

Stump, E., and Kumar, V. (2004), Workspace delineation of cable-actuated parallel manipulators, ASME Design Engineering Technical Conferences, Salt Lake City, UT, USA.

Tadokoro, S., Nishioka, S., Kimura, T., Hattori, M., Takamori, T., and Maeda, K. (1996), On fundamental design of wire configurations of wire-driven parallel manipulators with redundancy, Proceedings of the 1996 Japan/USA Symposium on Flexible Automation, Boston, MA, USA, pp. 151-158.

Takeda, Y., and Funabashi, H. (2000), Kinematic synthesis of spatial in-parallel wiredriven mechanism with six degrees of freedom with high force transmissibility, ASME Design Engineering Technical Conferences, Baltimore, MD, USA.

Verhoeven, R., and Hiller, M. (2000), Estimating the controllable workspace of tendonbased stewart platforms, in Advances in Robot Kinematics, Piran-Portoroz, Slovenia.

Verhoeven, R. (2004), Analysis of the workspace of tendon-based Stewart platforms, Ph.D. dissertation, Duisburg Essen University, Duisburg, Germany.

Williams II, R. L., Gallina, P., and Vadia, J. (2003), Planar translational cable-directdriven robots, Journal of Robotic Systems, vol. 20, no. 3, pp. 107-120. 